\title{
Electronic and Ionic Transport Dynamics in Organolead Halide Perovskites
}

Dehui Li ${ }^{1}$, Hao $W u^{2}$, Hung-Chieh Cheng ${ }^{2}$, Gongming Wang ${ }^{1,3}, Y u H u a n g^{2,3}$, and Xiangfeng $\operatorname{Duan}^{1,3 *}$

${ }^{1}$ Department of Chemistry and Biochemistry, University of California, Los Angeles, California 90095, USA.

${ }^{2}$ Department of Materials Science and Engineering, University of California, Los Angeles, California 90095, USA.

${ }^{3}$ California Nanosystems Institute, University of California, Los Angeles, California 90095, USA.

*Correspondence to: Email: xduan@chem.ucla.edu; 


\section{Simulation on evolution of the distribution of the ions}

Starting from the continuum theory developed in ionic liquids ${ }^{1}$, we have studied the evolution of the distribution of the ions under the applied bias with time. Previous studies indicate iodine vacancies dominate ion migration in our devices while migration rates of $\mathrm{Pb}$ and $\mathrm{MA}$ vacancies are orders of magnitude smaller than that of iodine vacancies. $^{2-5}$ Therefore, we only consider iodine vacancies as mobile ions and take the $\mathrm{Pb}$ and $\mathrm{MA}$ vacancies as stationary background during our simulation. An applied external bias would result in the redistribution of the ions and thus the local electrical potential. The local electrical potential would in turn drive the ion migration. In addition, the presence of ion gradient induced by the applied bias would lead to the ion diffusion. Therefore, the dynamics of ion migration can be described by a set of coupled partial differential equations which take into account the local electric field (modified Poisson equation), and ion drift and diffusion ${ }^{1}$ :

$$
\begin{aligned}
& \frac{1}{D c} \frac{\partial c}{\partial t}=2 v \frac{\partial^{2} c}{\partial x^{2}}+\frac{1}{v c}(1-v c-v B)(3 v c+v B-1) \frac{\partial^{2} u}{\partial x^{2}} \\
& l_{c}^{2} \frac{\partial^{4} u}{\partial x^{4}}-\frac{\partial^{2} u}{\partial x^{2}}-4 \pi l_{B}(c-B)=0
\end{aligned}
$$

where $D$ is diffusion coefficient, $c$ is the concentration of iodine vacancies, $v$ is the lattice volume, $B$ is the concentration of the $\mathrm{Pb}$ and MA vacancies, $u$ is local electric potential, $l_{c}$ is characteristic length for the short-ranged interactions and $l_{B}$ is the Bjerrum length. The first equation accounts for the ion drift and diffusion while the second one is the modified Poisson equation describing the local electrical field.

Similar to the ionic liquid case, we approximate our system to be an ionic solid with initially equal positive and negative ion concentration $B$ bounded by two parallel electrodes located at 0 and $\mathrm{L}$. A voltage of $3 \mathrm{~V}$ is applied at the time $\mathrm{t}=0$. To make the numerical calculation easier, we introduce dimensionless parameters: $r=v B, T=\left(D / L l_{D}\right) t$, $X=x / L$ and $C=c / B$. With those simplicities, the initial conditions can be described as

$$
\begin{aligned}
& C(X, 0)=1 \\
& u(X, 0)=3(1-X)
\end{aligned}
$$

The boundary conditions can be expressed as ${ }^{1}$ 


$$
\begin{aligned}
& u(0, T)=3, u(L, T)=0 \\
& \frac{\partial^{3} u}{\partial X^{3}}(0, T)=\frac{\partial^{3} u}{\partial X^{3}}(L, T)=0 \\
& {\left[\frac{4 \pi \sigma L}{\varepsilon}(1-r C-r) C+(1-r) \frac{\partial C}{\partial X}\right]_{X=0}=0} \\
& {\left[-\frac{4 \pi \sigma L}{\varepsilon}(1-r C-r) C+(1-r) \frac{\partial C}{\partial X}\right]_{X=L}=0}
\end{aligned}
$$

where $\sigma$ is the surface charge density and $\varepsilon$ is the dielectric constant of halide perovskite. While the first and second equations in (3) represent the constant potential conditions, the last two equations in (3) describe the no-flux conditions at the electrodes.

In the simulation, we take $r=0.25, \frac{4 \pi \sigma L}{\varepsilon}=5, L=20 \mathrm{~nm}, l_{c}=0.5 \mathrm{~nm}$ and $l_{B}=2.3$ $\mathrm{nm}$. The calculated evolution of spatial distribution of iodine vacancies is displayed in Fig. 2. Our simulation results indicate that the positive ions (iodine vacancies) pile gradually up close to cathode while net negative ions exist around the anode with time after the external bias is applied. Finally, an equilibrium condition is achieved that the maximum ion concentration is accumulated close the cathode area. The distribution of iodine vacancies gradually extends more and more into the channel with time, which can be attributed to the expelling of positive ions by the pre-accumulated positive ions close to the cathode similar to the ionic liquid case. ${ }^{1}$ 

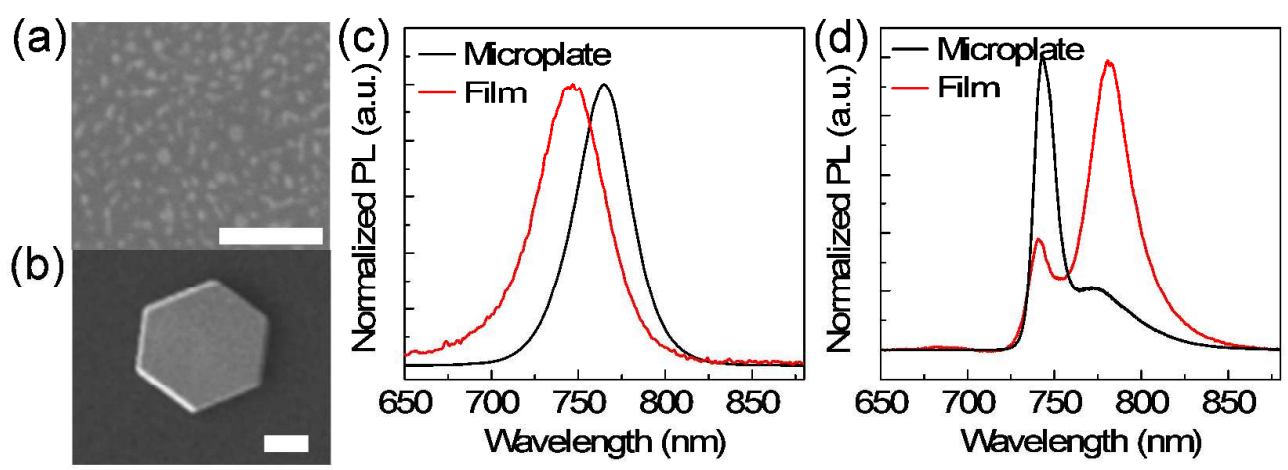

Figure S1. (a, b) The typical SEM images of perovskite film (a) and microplate (b). The scale bar is $1 \mu \mathrm{m}$. (c, d) Photoluminescence (PL) spectra of the perovskite film and microplates at room temperature (c) and at $77 \mathrm{~K}$ (d). Compared with perovskite microplate, the emission peak of perovskite film shows a blue shift, indicating the smaller crystal size of perovskite film. 


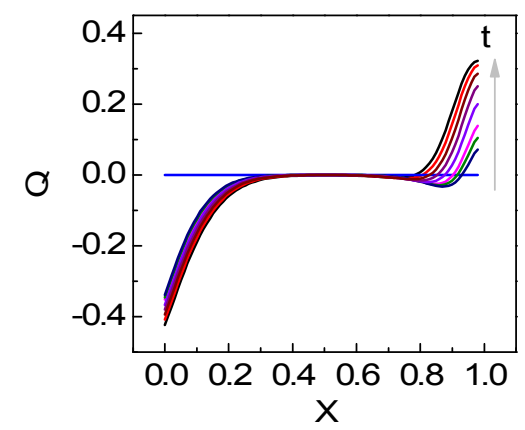

Figure S2. The calculated ion distribution and electric potential distribution across the organolead halide perovskite device channel. The calculated spatial distribution of iodine vacancies with time under an applied external bias of $3 \mathrm{~V}$. The curves from bottom to top represent the ion distribution with the elapse of time after the external bias is applied. 

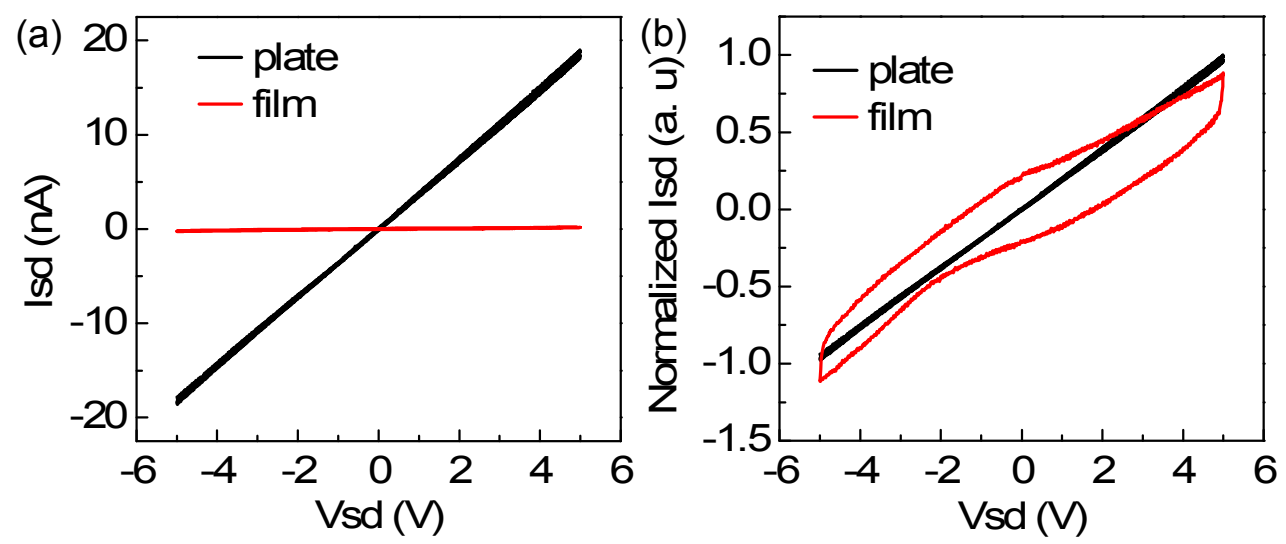

Figure S3. Comparisons of current-voltage curves in perovskite film and microplate devices. a, The IV curves for the organolead halide perovskite film and plate device under white light illumination (light power: $\sim 8 \mathrm{~mW}$ ). $b$, The normalized IV curves for the organolead halide perovskite film and plate device under white light illumination (light power: $\sim 8 \mathrm{~mW}$ ) in order to compare the hysteresis between them. 

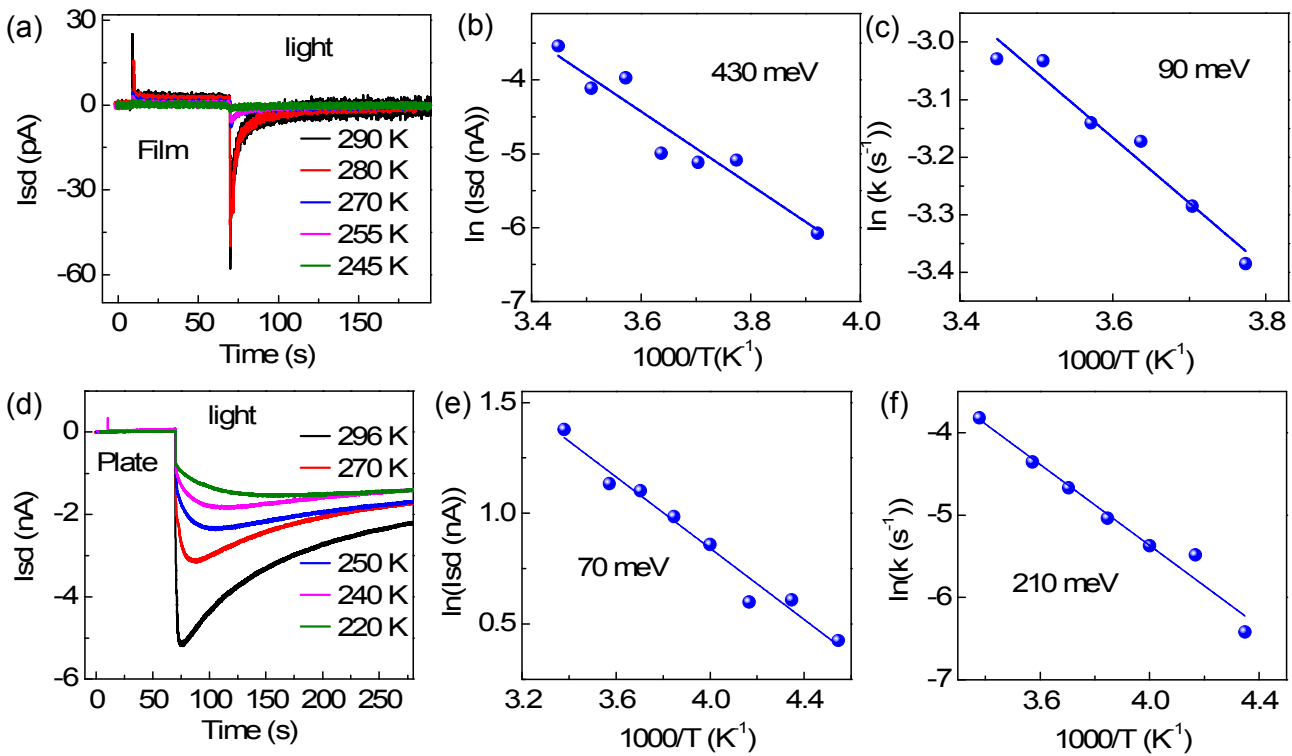

Figure S4. Comparisons of temperature dependent temporal response in organolead halide perovskite film and microplate devices under illumination at stage 2. a, The temperature dependence of temporal response curves under the applied external bias of $3 \mathrm{~V}$ in the halide perovskite film device under white light illumination (light power: $~ 8$ $\mathrm{mW} / \mathrm{cm}^{2}$ ). b. Arrhenius plot of the temperature dependence of $I_{2}$ extracted from (a). The solid line is linear fitting result. c, Arrhenius plot of the temperature dependence of ion decay rate $k_{2}$ extracted from (a). The solid line is linear fitting result. d, The temperature dependence of temporal response curves under the applied external bias of $3 \mathrm{~V}$ in a halide perovskite microplate device under white light illumination (light power: $\sim 8 \mathrm{~mW} / \mathrm{cm}^{2}$ ). e, Arrhenius plot of the temperature dependence of $I_{2}$ extracted from (d). The solid line are linear fitting result. $f$, Arrhenius plot of the temperature dependence of ion decay rate $k_{2}$ extracted from (d). The solid line is linear fitting result. 


\begin{tabular}{|l|l|l|l|}
\hline & $\mathrm{V}_{1}(\mathrm{eV})$ & $\mathrm{V}_{\mathrm{MA}}(\mathrm{eV})$ & $\mathrm{V}_{\mathrm{Pb}}(\mathrm{eV})$ \\
\hline Ref $1^{4}$ & $0.58(0.61)$ & 0.84 & 2.31 \\
\hline Ref $2^{5}$ & 0.08 & 0.46 & 0.80 \\
\hline Ref $3^{3}$ & $0.32 / 0.44$ & $0.57 / 0.89$ & -- \\
\hline Ref $4^{2}$ & $0.28 /(0.33)$ & 0.7 & 1.4 \\
\hline
\end{tabular}

Table S1. The summary of the activation energy from literatures. In ref 3 , the two values correspond to the different migration directions. The values in parentheses are experimental results while others are theoretical values. 


\section{References}

1. Lee, A. A.; Kondrat, S.; Vella, D.; Goriely, A., Dynamics of Ion Transport in Ionic Liquids. Phys. Rev. Lett. 2015, 115, 106101.

2. Meloni, S.; Moehl, T.; Tress, W.; Franckevičius, M.; Saliba, M.; Lee, Y. H.; Gao, P.; Nazeeruddin, M. K.; Zakeeruddin, S. M.; Rothlisberger, U., Ionic PolarizationInduced Current-Voltage Hysteresis in $\mathrm{CH}_{3} \mathrm{NH}_{3} \mathrm{PbX}_{3}$ Perovskite Solar Cells. Nat. Commun. 2016, 7, 10334.

3. Haruyama, J.; Sodeyama, K.; Han, L.; Tateyama, Y., First-Principles Study of Ion Diffusion in Perovskite Solar Cell Sensitizers. J. Am. Chem. Soc. 2015, 137, 1004810051.

4. Eames, C.; Frost, J. M.; Barnes, P. R. F.; O'Regan, B. C.; Walsh, A.; Islam, M. S., Ionic Transport in Hybrid Lead Iodide Perovskite Solar Cells. Nat. Commun. 2015, $6,7497$.

5. Azpiroz, J. M.; Mosconi, E.; Bisquert, J.; De Angelis, F., Defect Migration in Methylammonium Lead Iodide and Its Role in Perovskite Solar Cell Operation. Energy Environ. Sci. 2015, 8, 2118-2127. 